\title{
Closing the Gap: How Masculinity Affects Colorectal Cancer Screening in African-American Men
}

\author{
John M. Carethers ${ }^{1,2}$ \\ Accepted: 14 March 2021 / Published online: 3 April 2021 \\ (c) The Author(s), under exclusive licence to Springer Science+Business Media, LLC, part of Springer Nature 2021
}

In 2021 , there will be an estimated 284,000 breast cancers, 14,480 cervical cancers, 248,530 prostate cancers, and 154,860 colorectal cancers (CRCs) diagnosed in the USA, most of which can be detected at earlier stages by current screening modalities (e.g., mammography, Papanicolaou test, serum prostate-specific antigen (PSA) and rectal examination, fecal immunochemical test, fecal DNA test, or colonoscopy) in order to reduce death from the disease [1]. Indeed, mass cancer screening, the most effective method for averting cancer deaths, was a major factor in the prevention of over 3 million cancer deaths for men and women between 1991 and 2018 [1]. Focused education and other targeted screening enhancement strategies have been effective for gender-specific cancers such as breast, cervical, and prostate cancer, with $65 \%$ of eligible women receiving mammography within the past 2 years, $85 \%$ of eligible women being up to date with cervical cancer screening, and $37 \%$ of eligible men receiving a PSA within the past year [2]. CRC is a gender-neutral disease affecting both men and women nearly equally [1]. Compared with breast, cervical, and prostate cancer, CRC is the more deadly cancer, with an estimated 52,980 deaths projected for 2021 [1]. More screening tests have been devised for CRC than for any other cancer due to its deadly nature [3]. In $2015,65 \%$ of eligible men and women underwent CRC screening by one or more of the available modalities in the USA [2].

There is a marked disparity in CRC incidence, cancer stage, and cancer mortality by race, with African-Americans having the highest of these rates among any of the major racial and ethnic groups in the USA. [1-5]. The

John M. Carethers

jcarethe@umich.edu

1 Department of Internal Medicine, University of Michigan, 3100 Taubman Center, 1500 E. Medical Center Drive, Ann Arbor, MI 48109-5368, USA

2 Department of Human Genetics and Rogel Cancer Center, University of Michigan, Ann Arbor, USA
African-American:White incidence ratio for CRC is 1.13 , indicating that for every $100 \mathrm{CRCs}$ in Whites, there are 113 CRCs in African-Americans [5]. Similarly, the racial mortality ratio for CRC is 1.32 [5]. These ratios indicate not only a disparity for CRC incidence, but also an increased mortality disparity for among African-Americans following diagnosis, contributed in part by a more advanced cancer stage at presentation [1,2]. Moreover, the median age for CRC diagnosis for African-Americans is 3-7 years younger than for Whites, with the median age of 63 years for African-American men and 64 years for African-American women, compared with 66 years for White men and 70 years for White women [1, 6]. The root cause driving these race-based disparities for CRC is socioeconomic disadvantage, a factor that affects the lifetime cancer risk of an individual [7]. Overall generational structural disadvantages in the U.S. initiate a cascade of consequential lifelong effects that increase the risk for CRC, commencing with lower socioeconomic status of families and individuals that lessen the ability to obtain higher education and bears on factors such as the type of neighborhood, exposure to pollutants, and the amount of open park spaces available for physical activity. Other factors include limited employment opportunities and limited access to healthy foods despite increased exposure to tobacco and alcohol. Healthcare access and preventive services may also be limited. Over time, the contributions from an unhealthy diet, lack of physical activity, overuse of tobacco and alcohol, and limited access to preventive services alter the gut microbiome, increase localized tissue inflammation, increase the prevalence of obesity and metabolic disease, and compromise immunity, all increasing CRC risk [7].

These disparities assume great importance for achieving a high uptake for CRC screening in the AfricanAmerican population. Indeed, though screening for CRC is highly costeffective for the entire average-risk eligible U.S. population, it is the most cost-effective in the African-American population, compared with any other racial or ethnic group, due to the earlier age of presentation independent of the type of 
screening modality [6]. Yet, rates of overall screening uptake for AfricanAmericans lag behind the screening uptake for Whites by an average of 3-4\% [2]. Moreover, since positive non-colonoscopic CRC screening tests require a follow-up colonoscopy with potential biopsy and/or removal of lesions, an additional impediment exists, since AfricanAmericans are less likely than White Americans to undergo follow-up colonoscopy within 1 year of an abnormal screening test [6].

There are several likely barriers that contribute to the observed differences in screening utilization and follow-up between African-Americans and Whites [7]. Patient factors include lack of knowledge and education regarding the need and importance of screening, fear of diagnosis and fatalism, mistrust of aspects of the health system, differing insurance coverage, and an inability to fully undergo all of the processes involved with completion of screening. Provider factors include the lack of recommendation of CRC screening to African-American patients. Systemic factors include economic barriers such excessive out-of-pocket costs and the inability to successfully navigate health system processes in order to complete screening.

An intervention framework to address patient, provider, and systemic factors involves utilizing community and population outreach strategies combined with provider and teambased strategies, several of which increase CRC screening uptake [7]. One of the most potent interventions, assistance with healthcare navigation, eliminated CRC disparities between African-Americans and Caucasians, equalizing access to CRC screening despite of the many aforementioned pre-existing disparities according to a 2013 study [8]. Over a 9-year period, assistance with navigation of 10,000 average-risk patients increased CRC screening for both African-Americans from 47.8 to $73.5 \%$ and Whites from 58.0 to $74.7 \%$ while decreasing CRC incidence in AfricanAmericans from 68/100,000 to 48/100,000 and in Whites from $60 / 100,000$ to $48 / 100,000$, totally eliminating the CRC incidence disparity [8]. The disparity in CRC mortality was also reduced, with rates for African-American CRC deaths substantially reduced, approaching that of an also considerably lowered White rate at the end of the 9-year study [8]. Thus, it is clear that interventions that increase CRC screening rates can eliminate the observed disparities and lower the CRC incidence and mortality for the entire population.

National screening efforts have accelerated the lowering of CRC incidence and mortality over the past 20 years for African-Americans and Whites alike. Though the disparity gap has been slowly narrowing over this period with the uptick in screening rates in the overall population [2], the coronavirus disease 2019 (COVID-19) pandemic has decelerated that progress, with stark reductions in screening utilization and delay of preventive services [9]. Even relative short delays of cancer screening as brief as 6 months can eventuate in excess CRCs over the ensuing
10 years [9]. Unfortunately, the gains in reducing the disparity gap will most likely be undone, perhaps re-setting the gap to a point that existed 10 years ago. COVID-19 has also decreased screening utilization due to additional factors for patients, providers and the health system. Clearly understanding factors that might drive avoidance, as well as identifying factors that may drive completion of CRC screening are highly important in achieving compliance with screening that will reduce CRC incidence and mortality.

In this issue of Digestive Diseases and Sciences, Earl and colleagues sought to identify determinants that affect CRC screening behavior, focusing in particular on the influences of masculinity in order to inform awareness of the types of health messages that could be part of an intervention framework considering gender and cultural sensitivity [10]. A group of 32 self-identifying African-American men ages 45-75 years participated in semi-structured 30-min interviews conducted in person with a trained AfricanAmerican staff. A group of 103 enrollees recruited from primary care clinics and community centers participated in surveys addressing CRC knowledge testing, barriers to screening, masculinity effects on screening, and responses to $\mathrm{CRC}$ screening messaging. Among the group of 32 interviewed patients, 17 had been previously screened for CRC [10]. Major differences in beliefs for CRC were observed between previously screened and non-screened patients. For instance, most previously screened patients knew the age for commencing CRC screening, indicating that a healthcare provider was the most important screening motivator. About a third of the 32 patients described how being a male amplified the importance of holding responsibility for their families as a prioritizing factor for their own health. Among the 103 surveyed enrollees, $46.1 \%$ had been previously screened for CRC [10]. Previously screened patients scored higher on the CRC knowledge test (e.g., that CRC increases with age). Surprisingly, though barriers to screening among all surveyed were not perceived to be high, $48 \%$ agreed that a provider did not recommend screening. Those who were previously screened for CRC scored significantly higher regarding masculinity factors than those who were not $(57.4 \%$ vs $86.9 \%, P<0.01)$ [10]. Higher scored persuasive messages included "family depends on you" and "doing it to stay healthy".

The Earl et al. study suggests that contrary to general perception, traditional beliefs of masculinity can positively affect behavior for CRC screening. Use of this knowledge might be used persuade African-American men to initiate and complete CRC screening. Messaging can be tailored and strengthened by all providers recommending CRC screening to all eligible patients in order to help close the screening gap among African-American men. 
Acknowledgments Supported by the United States Public Health Service (R01 CA206010), the A. Alfred Taubman Medical Research Institute of the University of Michigan, and the Rogel Cancer Center of the University of Michigan. The funders had no role in study design, data collection and analysis, decision to publish, or preparation of the manuscript.

\section{Declarations}

Conflicts of interest No potential conflicts of interest are disclosed.

\section{References}

1. Siegel RL, Miller KD, Fuchs HE, Jemal A. Cancer statistics, 2021. CA Cancer J Clin 2021;71:7-33

2. DeSantis CE, Miller KD, Sauer AG, Jemal A, Siegel RL. Cancer statistics for African Americans, 2019. CA Cancer J Clin 2019;69:211-233

3. Carethers JM. Fecal DNA testing for colorectal cancer screening. Annu Rev Med 2020;71:59-69

4. Zavala V, Bracci PM, Carethers JM, Carvajal-Carmona L, Coggins NB, Cruz-Correa MR, Davis M, de Smith AJ, Dutil J, Figueiredo JC, Fox R, Graves KD, Gomez SL, Llera A, Neuhausen SL, Newman L, Nguyen T, Palmer JR, Palmer NR, Perez-Stable EJ, Piawah S, Rodriquez EJ, Sanabria-Salas MC, Schmit SL, Schmidt SL, Serrano-Gomez SJ, Stern MC, Weitzel J, Yang JJ, Zabaleta J,
Ziv E, Fejerman L. Cancer health disparities in US racial/ethnic minorities. Br J Cancer 2021;124:315-332

5. Ashktorab H, Brim H, Kupfer SS, Carethers JM. Racial disparity in gastrointestinal cancer risk. Gastroenterology 2017;153:910-923

6. Carethers JM. Screening for colorectal cancer in African Americans: determinants and rationale for an earlier age to commence screening. Dig Dis Sci 2015;60:711-721

7. Carethers JM, Doubeni CA. Causes of socioeconomic disparities in colorectal cancer and intervention framework and strategies. Gastroenterology 2020;158:354-367

8. Grubbs SS, Polite BN, Carney J Jr, Bowser W, Rogers J, Katurakes N, Hess P, Paskett ED. Eliminating racial disparities in colorectal cancer in the real world: it took a village. J Clin Oncol 2013;31:1928-1930

9. Carethers JM, Sengupta R, Blakey R, Ribas A, D'Souza G. Disparities in cancer prevention in the COVID-19 era. Cancer Prev Res 2020;13:893-896

10. Earl V, Beasley D, Ye C, Halpin SN, Gauthreaux N, Escoffery NC, Chawla S. Barriers and facilitators to colorectal cancer screening in African American men. Dig Dis Sci. (Epub ahead of print). https://doi.org/10.1007/s10620-021-06960-0.

Publisher's Note Springer Nature remains neutral with regard to jurisdictional claims in published maps and institutional affiliations. 